\title{
A PACKET LOSS ESTIMATION MODEL AND ITS APPLICATION TO RELIABLE MESH- BASED P2P VIDEO STREAMING
}

\author{
Chi-Wen Lo ${ }^{1}$, Chia-Wen Lin ${ }^{1}$, Yung-Chang Chen ${ }^{1}$, and Jen-Yu Yu ${ }^{2}$ \\ ${ }^{1}$ Department of Electrical Engineering, National Tsing Hua University, Hsinchu, Taiwan \\ ${ }^{2}$ Information and Communications Research Labs, Industrial Technology Research Institute, Taiwan
}

\begin{abstract}
This paper proposes a model to estimate the packet loss probability in a mesh-based P2P network. Because of the irregular mesh structure, packet loss estimation for a mesh-based P2P network is more complicated than that in a tree-based network. The proposed model takes into account the channel packet drop rate, peer dynamics, and FEC protection to capture the heterogeneous packet loss behavior of individual video substreams transmitted over the irregular transmission paths of a mesh network. The simulation results show that the proposed packet loss model can accurately estimate the packet loss in a mesh-based P2P network. Based on the proposed model, we also propose a peer selection mechanism which can effectively mitigate packet loss propagation by selecting at a parent-peer the candidate child-peers that can achieve the minimal packet loss probability compared to others, to transmit the FEC redundant substream.
\end{abstract}

Index Terms - Error protection, P2P video streaming system, peer selection, FEC

\section{INTRODUCTION}

Due to the fast growing deployment of advanced network and multimedia technologies, video streaming services are able to provide stable quality. The key of a successful video streaming system lies in the video quality perceived by users. One of the major challenges to video streaming services is packet loss. Since the IP-based networks only support best effort services, video packets are not well protected. If a video packet cannot be received before its playback time, the reconstructed video quality may be seriously damaged.

Packet-level FEC has been often utilized to protect video packets in video streaming systems, where the channel encoder, such as Reed-Solomon code, encodes the video bitstreams into $k$ data packets and extra $n-k$ redundant packets, denoted as $F E C(n, k)$. The receiver can fully reconstruct the original signal if at least any $k$ out of $n$ packets are received. On the other hand, $F E C(n, k)$ scheme can tolerate $n-k$ packets loss at most. The FEC protection capability can be increased by increasing $n$ to ensure enough packets can be received. Compared with retransmission-based schemes in which the receiver requests the re-transmissions for the lost packets, the FEC-based scheme is more suitable for timesensitive video applications, such as real-time video streaming.

The method proposed in [1] applies FEC to recover packet loss in an overlay streaming system. The FEC codes are decided according to the channel conditions of the segments of a delivery path but the peer dynamics are not considered. The performance of FEC codes with different video frame types was analyzed in [2]. Due to the motion prediction structure of video encoder, I and $\mathrm{P}$ frames are more important than B frames. The results in [2] show that the packet loss probability in the $\mathrm{P} 2 \mathrm{P}$ video streaming system can be reduced by unequal error protection (UEP). With UEP, the important video frames are assigned to more redundancy to overcome packet loss. Multiple-description FEC [3] combines UEP and layer video coding to provide a robust service among heterogeneous peers. In [4], the packet loss probability and packet loss accumulation in a multi-source tree-based $\mathrm{P} 2 \mathrm{P}$ system are analyzed.

The packet loss estimation for $\mathrm{P} 2 \mathrm{P}$ networks is more complicated than that for traditional client-server structures. Because the video sources are the multiple peers rather than a single server, the packet loss propagates through the inter-peers transmissions. Moreover, peers will usually unexpectedly join and leave the systems. An analytic model proposed in [4] can be used to estimate the packet loss probability of the candidate child-peers in tree-based P2P networks. However, the model cannot be directly applied in mesh structures. In a tree-based P2P network, each peer is located in a specific depth in the tree. Therefore, the parent peers of each peer have the same packet loss accumulation if the channel drop rates between peers are homogeneous. In a mesh-based P2P network, however, the peers are randomly located in the irregular mesh structure. As a result, the packet loss accumulation from the multiple parent peers is heterogeneous so that the tree-based packet loss model cannot characterize the packet loss well in the meshbased P2P network. Since, many popular P2P streaming systems, such as CoolStreaming [5], PPStreaming [6], and PPLive [7] are mesh-based structures, an accurate mesh-based packet loss model is desirable.

To the best of our knowledge, the error propagation of P2P video streaming in a mesh-based network was rarely addressed because of the network's irregular structure. In this paper, we propose a model to estimate the packet loss probability in a meshbased P2P network. The proposed model takes into account the channel packet drop rate, peer dynamics, and FEC protection to capture the heterogeneous packet loss behavior of individual video substreams transmitted over the irregular transmission paths of a mesh network. Based on the proposed model, we also propose a peer selection mechanism which can effectively mitigate packet loss propagation by selecting at a parent-peer the candidate childpeers that can achieve the minimal packet loss probability compared to others, to transmit the FEC redundant substream. As a result, the packet loss caused by peer departure, the absent packets in the parent peers, and the channel packet drop rate, are effectively mitigated. 
The rest of this paper is organized as follows. The framework of FEC-based error protection for push-pull P2P video streaming is presented in Section 2. The proposed packet loss models are described in Section 3. In Section 4, the peer selection method is presented. Section 5 shows the simulation settings and the results. Finally, conclusions are drawn in Section 6.

\section{FEC-BASED ERROR PROTECTION FOR P2P VIDEO STREAMING}

Fig. 1 depicts the packetization method used in this paper. We assume that each group of pictures (GOP) is encoded with the same bitrate and with the same number of video packets. Then, the $k$ video source packets of each GOP are encoded with $F E C(n, k)$ code to generate additional $n-k$ redundant packets. The corresponding packets of the GOPs compose the video substreams. As shown in Fig. 1, video substream 1 contains the first packets from GOP \#1 and \#N. During a streaming session, the child-peers subscribe to their parent peers for the video substreams (i.e., a pull process). Once the parent-peers accept the subscription, the parentpeers continuously push the corresponding packets to their child peers, as known as the push-pull methods [5].

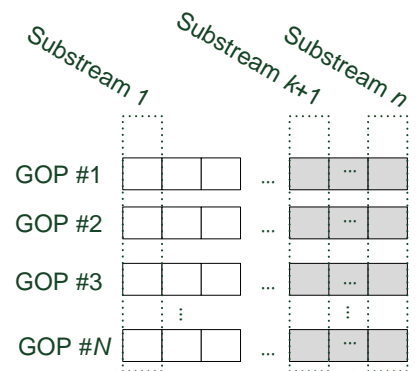

Fig. 1. Data protection with $F E C(n, k)$, where white blocks indicate the data packets and the grey ones indicate FEC redundant blocks.

\section{PROPOSED MESH-BASED PACKET LOSS MODELS}

\subsection{Packet Loss Accumulation in Tree-based and Mesh-based P2P Structures}

The error propagation behavior due to packet loss in a meshbased P2P streaming system is significantly different from that for a tree-based system. Fig. 2(a) shows an example of tree-based topology where each peer is located at a specific depth of a tree. In such a topology, a peer's parent-peers which are at the same depth will contribute the same degree of channel packet loss. For example, the parent-peers of peer \#10 are located at depth 2, and they all receive the video substreams from the server (root) through transmission of two hops. Hence, each substream sent to peer \#10 has the same packet loss probability accumulated along the links from the server to peer \#10 should the packet drop rates of individual links be homogeneous.

Consider the mesh-based topology shown in Fig. 2(b). Due to peers' self-organized behavior, there is no regular structure that can completely describe the peer interconnections of a mesh network. Since the peers are randomly located in the mesh network, substreams may contribute significantly different levels of channel packet loss. As depicted in Fib. 2(b), for peer \#8, the substream received from peer \#1 passes through two hops from the server, whereas the substream obtained from peer \#5 passes through three hops. The accumulated packet loss probabilities of these two substreams are therefore not identical. Hence, the estimation of packet loss rate for cases involving multiple sources with heterogeneous packet loss rates is significantly more complicated than that for cases involving homogeneous sources. Consequently, we need a sophisticated model rather than a simple tree-based model to accurately characterize the error propagation behavior due to packet loss in an irregular mesh-based network.

\subsection{The Packet Loss Models of a Peer}

In the $\mathrm{P} 2 \mathrm{P}$ video streaming systems, the video packets are lost because of 1) Peer departure: When the parent peers leave the system, their child peers are no longer to receive the streaming packets. the burst packet loss is alleviated until the child peers find the replacement parent peer. 2) Channel packet loss: the video packets are dropped during the transmission. 3) Absent packets in the parent peer: the child peers cannot obtain the packets from their parent peers because the parent peer loses the video packets. By using FEC-based packet protection mechanism, the lost packets can be recovered. In what follows, we derive the packet loss probability models, which are based on the tree-based packet loss models [4], for each peer in the mesh-based P2P networks.

The packet loss probability that peer $x$ receives $n_{x}$ substreams can be expressed by

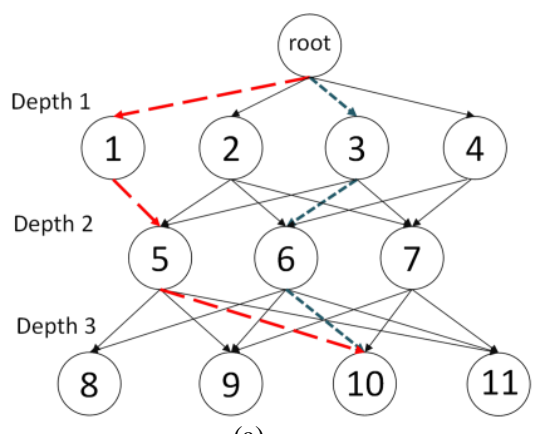

(a)

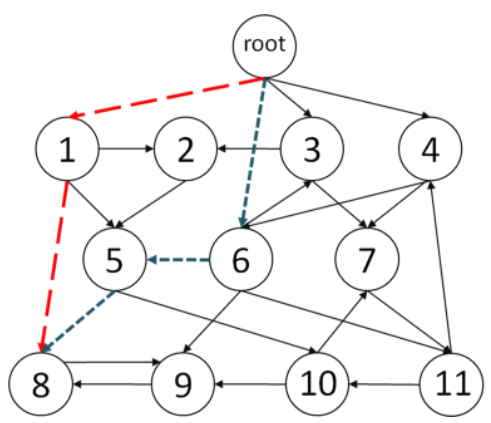

(b)

Fig. 2. Two examples of P2P video streaming structures: (a) a tree-based network topology, and (b) a mesh-based topology. The red dash lines and blue dot-dash line indicate two different substreams, whereas the solid lines indicate the other substreams. 


$$
Q_{x}\left(n_{x}\right)=\sum_{i=0}^{n_{x}} P_{i}^{n_{x}} \cdot q_{x, i}
$$

where $P_{i}^{n_{x}}$ is the probability of $i$ parent-peers leaving, which can be modeled by $n_{x}+1$ states Continuous-Time Markov Chain (CTMC) [4], and $q_{x, i}$ is the packet loss probability due to the leaving of $i$ parent-peers. The calculation of $q_{x, i}$ is described below.

When the number of leaving parent-peers exceeds $k$ (i.e., $i>$ $\left.n_{x}-k\right)$, the lost packets cannot be recovered. A packet gets lost due to either of the three events: 1) the parent-peer owning the packet has leaved the system, 2) the live parent-peer of the substream does not have the packet, and 3) the live peer has the packet, but the packet is dropped during the transmission. These conditions are expressed by

$$
\begin{aligned}
& q_{x, i>n_{x}-k}= \\
& \underbrace{\frac{i}{n_{x}}}_{\text {event } 1}+\underbrace{\left(\frac{n_{x}-i}{n_{x}} \cdot Q_{x}^{p}\right)}_{\text {event } 2}+\underbrace{\left(\frac{n_{x}-i}{n_{x}}\right) \cdot\left(1-Q_{x}^{p}\right) \cdot d_{x}^{p}}_{\text {event 3 }},
\end{aligned}
$$

where the three terms on the right-hand-side of (2) represent the occurrence probabilities of the three events, respectively.

In (2), $Q_{x}^{p}$ denotes the average packet loss probability of the substreams in peer $x$ 's parent-peers, which can be calculated by

$$
Q_{x}^{p}=\frac{1}{n_{x}} \sum_{j=0}^{n}\left\{S I_{x}(j) \cdot Q_{y}^{S_{-}}\right\}
$$

where $S I_{x}(j)$ is the substream indicator function, with $S I_{x}(j)=1$ indicating peer $x$ subscribes substream $j$, and $S I_{x}(j)=0$ indicating no subscription of substream $j$. Note, $\sum_{j=0}^{n} S I_{x}(j)=n_{x} . Q_{y}^{s_{-} j}$ is the packet loss probability of substream $j$ for parent peer $y$.

The average packet drop rate of the channels between peer $x$ and its parent peers can be computed by

$$
d_{x}^{p}=\frac{1}{n_{x}} \sum_{j=0}^{n}\left\{S I_{x}(j) \cdot d_{y}^{s_{-} j}\right\}
$$

where $d_{y}^{s_{-} j}$ is the packet drop rate of the channel to transmit substream $j$ whose parent peer is peer $y$.

As shown in (2), when the number of leaving peers $i \leq n_{x}-k$, the lost packets may be recovered. However, the lost packets caused by the parent-peer departure are not recoverable, should the number of received packets from the $n_{x}-i$ surviving live peers be less than $k$. This packet loss probability $C_{1}$ can be calculated by

$$
\begin{gathered}
C_{1}=\frac{i}{n_{x}} \cdot \sum_{w=0}^{k-1}\left(\begin{array}{c}
n_{x}-i \\
w
\end{array}\right) \cdot\left\{\left(1-Q_{x}^{p}\right)\left(1-d_{x}^{p}\right)\right\}^{w} . \\
\left\{1-\left(1-Q_{x}^{p}\right)\left(1-d_{x}^{p}\right)\right\}^{n_{x}-i-w} .
\end{gathered}
$$

Besides, a lost packet caused by the event that the live parent peer does not have the packet is not recoverable, if the number of received packets from the surviving live peers other than peer $x$ is less than $k$. This packet loss probability $C_{2}$ can be calculated by

$$
\begin{aligned}
C_{2}= & \left(\frac{n_{x}-i}{n_{x}} \cdot Q_{x}^{p}\right) \cdot \sum_{w=0}^{k-1}\left(\begin{array}{c}
n_{x}-i-1 \\
w
\end{array}\right) \cdot\left\{\left(1-Q_{x}^{p}\right)(1-\right. \\
& \left.\left.d_{x}^{p}\right)\right\}^{w} \cdot\left\{1-\left(1-Q_{x}^{p}\right)\left(1-d_{x}^{p}\right)\right\}^{n_{x}-i-1-w} .
\end{aligned}
$$

Finally, a lost packet getting dropped during the transmission cannot be recovered, if the number of received packets from the surviving live peers other than peer $x$ is less than $k$. This loss probability $C_{3}$ can be calculated by

$$
\begin{aligned}
C_{3}= & \left\{\frac{n_{x}-i}{n_{x}} \cdot\left(1-Q_{x}^{p}\right) \cdot d_{x}^{p}\right\} \cdot \sum_{w=0}^{k-1}\left(\begin{array}{c}
n_{x}-i-1 \\
\mathrm{w}
\end{array}\right) . \\
& \left\{\left(1-Q_{x}^{p}\right)\left(1-d_{x}^{p}\right)\right\}^{w} \cdot\left\{1-\left(1-Q_{x}^{p}\right)(1-\right. \\
& \left.\left.d_{x}^{p}\right)\right\}^{n_{x}-i-1-w} .
\end{aligned}
$$

As a result, $q_{y, i \leq n_{x}-k}$ is obtained by

$$
q_{y, i \leq n_{x}-k}=C_{1}+C_{2}+C_{3}
$$

\subsection{Error Propagation due to the Loss of a Substream}

In this section, we derive the packet loss probability models for the substreams received from the parent peers to characterize the heterogeneous packet loss over the various substream transmissions.

Note, when peer $x$ receives substream $j$ from peer $y$, the packets of substream $j$ may have already been lost by peer $y$ with a probability of $Q_{y}^{s_{-} j}$. Moreover, the probability that peer $y$ keeps staying in the system or leaving the system is $P_{0}^{1}$, and $P_{1}^{1}$, respectively. Therefore the mean packet loss probability of substream $j$ in peer $y$ is $P_{0}^{1} \cdot Q_{y}^{s_{-} j}+P_{1}^{1} \cdot 1$. In the case that peer $y$ owns the packets of substream $j$ and the packets are dropped during transmission with probability $d_{y}^{s_{-} j}$, the packet loss probability that peer $x$ receives substream $j$ from peer $y$ without FEC protection becomes

$$
C_{4}=1-\left\{1-\left(P_{0}^{1} \cdot Q_{y}^{s_{-} j}+P_{1}^{1} \cdot 1\right)\right\} \cdot\left(1-d_{y}^{s_{-} j}\right)
$$

A lost packet of substream $j$ cannot be recovered, if the number of packets received from the remaining $n_{x}-1$ substreams is not enough for FEC recovery. The probability of such event can be calculated by

$$
\begin{gathered}
C_{5}=\sum_{i=0}^{n_{x}-1} P_{i}^{n_{x-1}} \cdot\left\{\sum_{w=0}^{k-1}\left(\begin{array}{c}
n_{x}-1-i \\
w
\end{array}\right)((1-\right. \\
\left.\left.Q_{x}^{p \notin j}\right)\left(1-d_{x}^{p \notin j}\right)\right)^{w}(1- \\
\left.\left.\left(1-Q_{x}^{p \notin j}\right)\left(-d_{x}^{p \notin j}\right)\right)^{n_{x}-1-i-w}\right\} .
\end{gathered}
$$

where $Q_{x}^{p \notin j}$ denotes the average packet loss probability of the remaining substreams in parent-peers except peer $y$, and $d_{x}^{p \notin j}$ is the average packet drop rate of the channels to the parent-peers except peer $y$.

As a result, the packet loss probability of substream $j$ after FEC recovery is

$$
Q_{x}^{s_{-} j}=C_{4} \cdot C_{5}
$$

Then the child peers of peer $x$ can estimate their packet loss probability for the peers and for their substreams with the probability $Q_{x}^{S_{-} j}$.

\section{CONTRIBUTION-GUIDED PEER SELECTION}

In the proposed contribution-guided peer selection scheme, when a candidate peer requests a redundant substream from a parent-peer, the parent-peer will evaluate the candidate peer's 


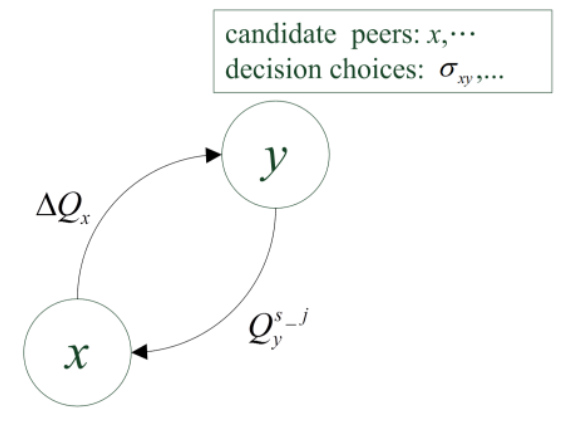

Fig. 3. Proposed peer selection process. The candidate child-peer $x$ estimates $Q_{y}^{s j}$ to derive the packet loss gain $\Delta Q_{x}$. Parent-peer $y$ collects the packet loss gains from all candidate child-peers and selects the appropriate peers accordingly.

contribution of forwarding the substream to its succeeding childpeers that also request the substream. To this end, we estimate the packet loss reduction contributed by a candidate peer. The more the packet loss reduction, the higher the candidate peer's contribution. As shown in Fig. 3, since peer $y$ is a parent-peer, the packet loss occurring in peer $y$ will propagate to its child-peer $x$ as well.

In order to reduce the packet loss, peer $x$ subscribes additional redundant substreams. Peer $x$ can send the subscription message to parent-peer $y$. Next, peer $x$ is added into the candidate set $\overline{\mathbf{C}}_{y}$ of peer $y$. However, since the uplink bandwidth of peers is limited, the peers have to efficiently allocate their limited bandwidth resource to achieve better streaming performance. Therefore, we should choose the child-peers who can further reduce the packet loss by assigning the redundant substream. Based on the proposed packet loss model, those "low-contribution" candidate peers whose subscription cannot further recover the packet loss will be rejected. Let $n_{x}+\sigma_{x y}$ be the total number of substreams expected to be received by peer $x$, where $\sigma_{x y}$ is the peer selection choice for candidate peer $x$. If peer $y$ accepts peer $x$ as child-peer, $\sigma_{x y}=1$; otherwise, $\sigma_{x y}=0$. We use the set of variables $\bar{\sigma}_{y}=\left\{\sigma_{x y} \mid x \in \overline{\mathbf{C}}_{y}\right\}$ to represent the set of peer selection choices.

The parent-peer $y$ selects the child-peers every peer selection period. During the peer selection period, the peers subscribing to peer $y$ are added into the candidate sets of peer $y$. Besides, the child-peers which have supported the redundant substreams and connected more than $T$ seconds are added into the candidate set as well. On the other hand, the connection time of video substreams is guaranteed at least $T$ seconds. The peer selection metric can be to minimize the total packet loss probability of all candidate peers through (12).

$$
\begin{aligned}
& \underset{\bar{\sigma}_{y}}{\arg \min } \sum_{x \in \bar{C}_{y}} Q_{x}\left(n_{x}+\sigma_{x y}\right) \\
& \text { subject to } \sum_{x \in \bar{C}_{y}} \sigma_{x y} \cdot R_{j} \leq U_{y}
\end{aligned}
$$

where $\sigma_{x y}=\{1,0\}, R_{j}$ is the video encoding rates of substream $j$, and $U_{y}$ is the available uplink bandwidth of peer $y$. Equation (12) is equivalent to

$$
\begin{aligned}
& \underset{\bar{\sigma}_{y}}{\arg \max } \sum_{x \in \bar{C}_{y}}\left(\sigma_{x y} \cdot \Delta Q_{x}\right) \\
& \text { subject to } \sum_{x \in \bar{C}_{y}} \sigma_{x y} \cdot R_{j} \leq U_{y}
\end{aligned}
$$

where $\Delta Q_{x}=Q_{x}\left(n_{x}\right)-Q_{x}\left(n_{x}+\sigma_{x y}\right)$ is the packet loss reduction after receiving the substream $j, Q_{x}^{p}$ and $d_{x}^{p}$ are updated by considering the new substream. This optimization problem is a $0 / 1$ knapsack problem that can be solved by dynamic programing.

\section{SIMULATION RESULTS}

We used P2Pstrsim [7] to evaluate the performance of the proposed method. In our simulation, the peers are self-organized into a mesh structure. The simulation time is set to 30 mins. We encoded a CIF $(352 \times 288)$ video at $30 \mathrm{fps}$ with a bitrate of $300 \mathrm{kbps}$ by the JM14.2 SVC coder [9]. The video bitstream is transmitted over mesh-based P2P networks, and the video packet loss rates are measured to evaluate the performance of proposed model and peer selection method.

First, we evaluate the accuracy of the packet loss estimation models. In Fig. 4, we compare the three packet loss rates: 1) the actual average packet loss rate measured from the received substreams at the receiving peers, denoted as "actual," 2) the average packet loss rate estimated by the proposed model, denoted as "M-model," and 3) the average packet loss rate estimated by the tree-based model, denoted as "T-model." However, since the treebased model cannot be directly applied in a mesh-based P2P network, we use the concept that the packet loss accumulation of each substream in a peer is identical. Therefore the $Q_{y}^{s_{-} j}$ in (2) is modified to $Q_{y}$, i.e., the estimated packet loss probability of peer $y$.

Fig 4(a) shows the packet loss accumulation of 1,000 peers without FEC protection and peer dynamics. In this simulation, a video bitstream is divided into 4 substreams. The average packet

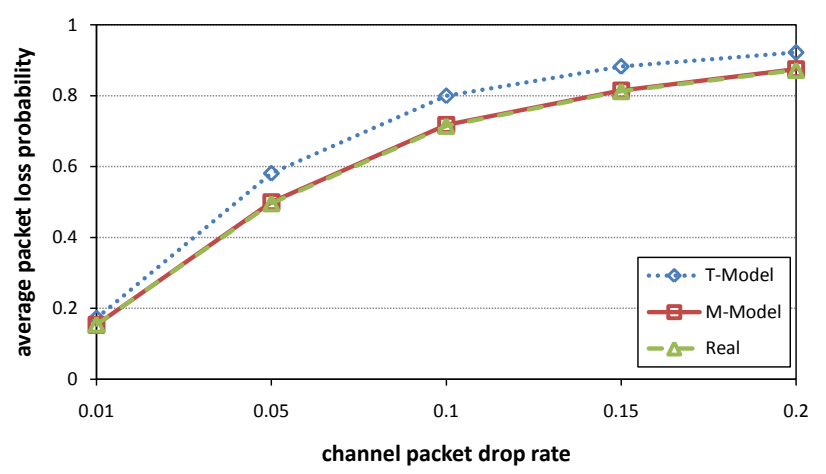

(a)

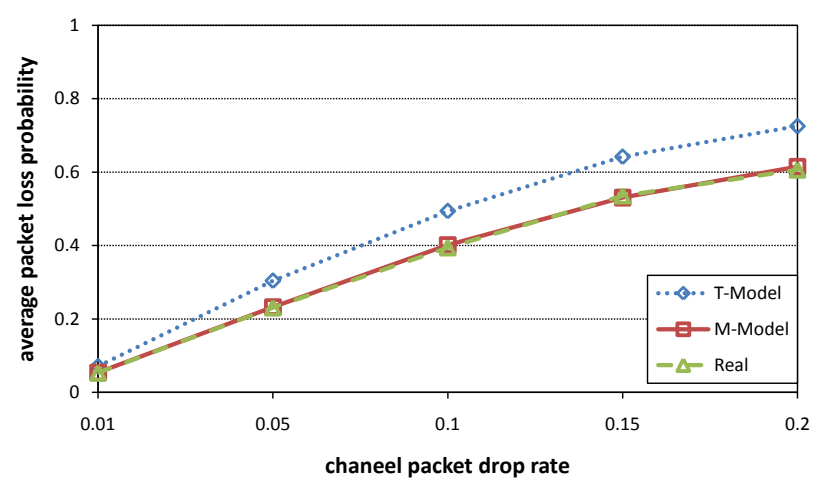

(b)

Fig. 4. Packet loss accumulation estimation for different-size P2P networks: (a) a network of 1000 peers, and (b) a network of 200 peers. 
loss rate increases with the channel packet drop rates. Under a low channel drop rate (e.g., 1\%), both the T-model and M-model can do a good job in the packet loss estimation. Nevertheless, when the channel packet drop rate increases to $5 \%, 10 \%, 15 \%$, and $20 \%$, compared with the actual loss rates, the T-model significantly overestimates the packet loss rate by $0.084,0.121,0.109$, and 0.066 , respectively. In contrast, the proposed M-model achieves fairly good estimation accuracy for various test cases under different network sizes and channel conditions. Similar observation can also be drawn from a test case with a much smaller network size of 200 peers, as shown in Fig. 4(b). Note, Fig. 4 shows that the average packet loss rates in the 200-peer network is lower than that in the 1,000-peer network. For a larger-size mesh-based P2P network, a video substream usually traverses more transmission hops from a video server to a receiver, leading to higher packet loss rate as the packet loss will accumulate along the hops.

Next we take into account peer dynamics and FEC protection in the model evaluation for a network of 1000 peers. Assume the 1,000 peers uniformly join the network within 30 minutes, and then the peers are leaving the system independently after certain viewing time that is assumed to be uniformly distributed in the range of 15 45 minutes. We also assume each user records the number of parent-peers leaving in each period of time and the time for finding a new replcaement parent-peer for calculating parameter $\rho$ for the CTMC model [4].

Fig 5(a) shows the packet loss estimation results with $F E C(5,4)$ protection. Under the low channel packet drop rates of $1 \%$ $9 \%$, since many lost packets can be recovered, the packet loss rate is lower than 0.05. However, the peers experience serious packet loss with a loss rate of 0.85 (or above) when the channel packet drop exceeds $10 \%$, due to the insufficient protection capability of $F E C(5,4)$ in coping with high channel drop rates and peer dynamics. In this case, the M-model still does a good job in the packet loss estimation except for the channel packet drop rates (around 10\% in this case) that result in a sharp transition of packet loss rates. The sharp transition occurs at the borderline of packet drop rate that $F E C(5,4)$ can protect against. Fig 5(a) shows that both the M-model and T-model tend to reflect the transition smoothly, thereby underestimating the packet loss rate. However, the M-model still much better estimation accuracy compared to the T-model. Fig 5(b) depicts the packet loss estimation results with $F E C(6,4)$ protection, showing that $F E C(6,4)$ can effectively recover the lost packets for the channel drop rates lower than $20 \%$. When the packet drop rate reaches $20 \%$, the M-model still estimates the packet loss accurately, whereas the T-model underestimates the packet loss rate by about 0.161 .

To evaluate the performance of our method in a heterogeneous network, five different classes of channel packet loss rates were applied: [0.01, 0.05], [0.01, 0.1], [0.01, 0.15], [0.01, $0.2]$, and $[0.01,0.25]$. The channel packet loss rate of each channel class is set to be uniformly distributed in the setting range, e.g., for the class $[0.01,0.05]$ the channel packet loss rates are uniformly distributed in $[0.01,0.05]$. Fig. 6 shows the packet loss estimation results with different FEC codes in the heterogeneous network. In Fig. 6(a), the simulation conditions are the same as those in Fig. 4(a) except the heterogeneous packet loss rates. The simulation results are consistent with Fig 4(a) in which the higher channel packet loss rates cause higher packet loss probability. The simulation conditions and results in Fig 6(b) are similar to Fig 5(b) in which FEC can be used to recover the packet loss in low channel packet loss rates. The results show that the M-Model still achieves accurate estimation results for heterogeneous channel conditions.

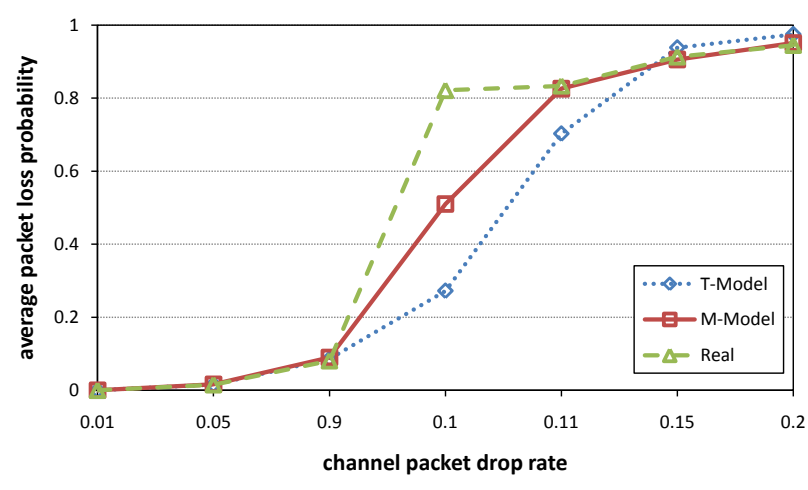

(a)

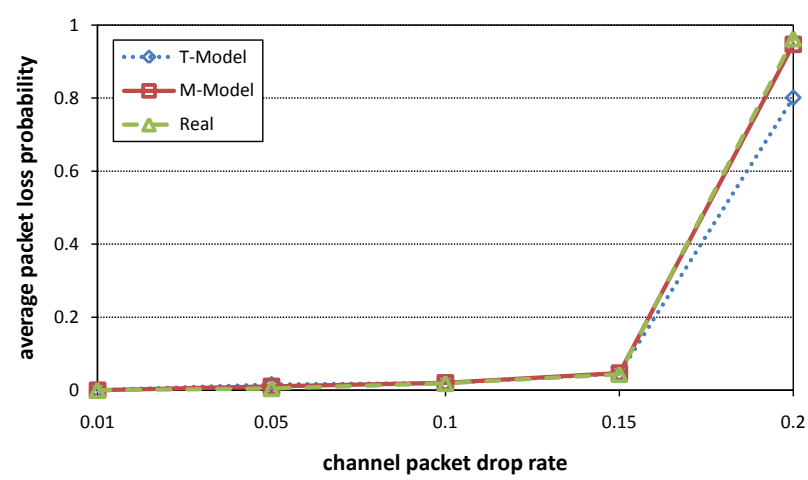

(b)

Fig. 5. Packet loss estimation with different FEC codes: (a) $F E C(5,4)$ and (b) $\operatorname{FEC}(6,4)$.

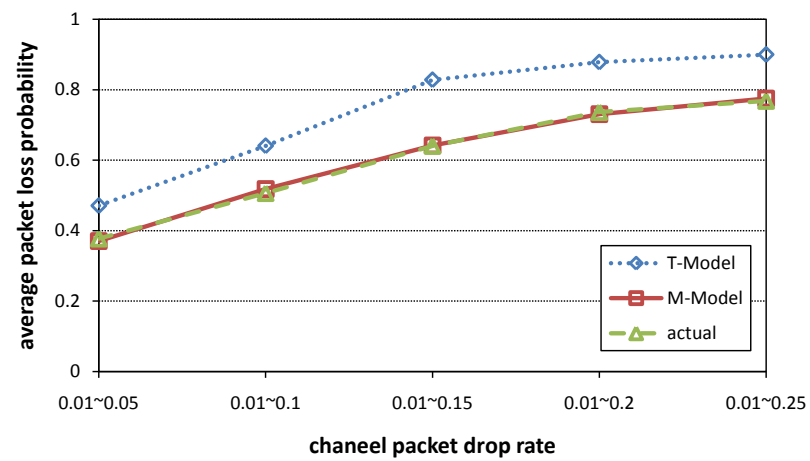

(a)

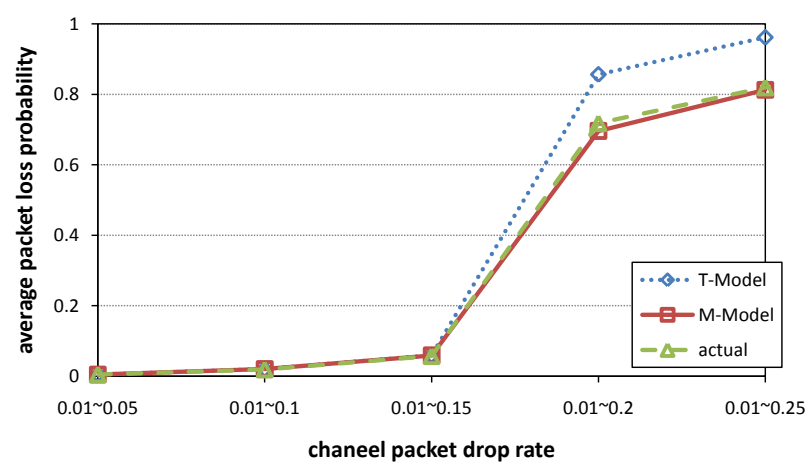

(b)

Fig. 6. Packet loss estimation for a heterogeneous network with different FEC codes: (a) $F E C(4,4)$ and (b) $F E C(5,4)$. The network involves 1000 peers under five classes of channel conditions. 


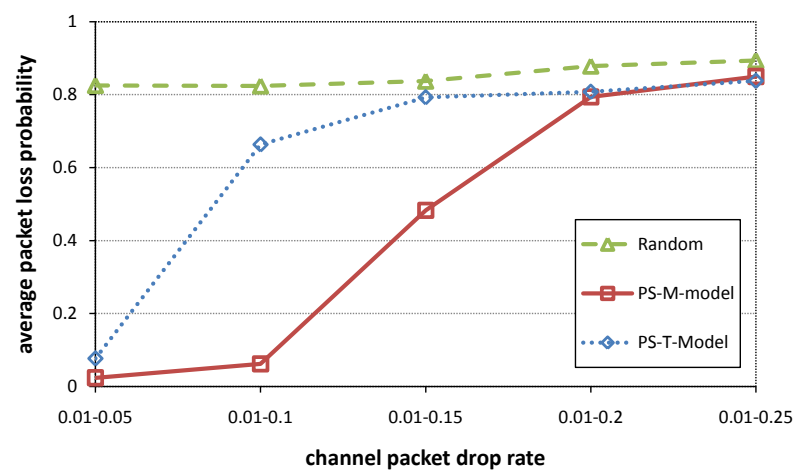

Fig. 7. Performance evaluation for three peer selection methods for a heterogeneous network with five classes of channel conditions. The three methods include random selection, selection based on the M-model, and selection based on the T-model.

The above simulation results demonstrate that the M-model achieves fairly good performance of packet loss estimation for mesh-structure P2P networks. In contrast, the T-model leads to significant underestimates of packet loss rate for the channel conditions with transition channel drop rates. This is because the M-model takes into account the packet loss of each substream in each transmission hop, whereas the T-model does not consider the heterogeneous packet loss among the substreams in a mesh-based P2P network.

Next, we evaluate the performances of peer selection methods for a P2P network with a limited peer uplink bandwidth that cannot fully support the total bandwidth demand of video substreams. In this simulation, $F E C(6,4)$ which generates $50 \%$ redundancy is applied, therefore the up-link bandwidth demand is at least 450 kbps. Three peer selection methods are compared: 1) random peer selection (denoted as "random"), where the parent-peers accept the substream subscriptions until their available uplink bandwidth is exhausted, 2) peer selection by M-model (denoted as "PS-Mmodel") where the parent-peers select the child-peers according to (7), and 3) peer selection by T-model, denoted as "PS-T-model."

Fig. 7 compare the performance of the three peer selection schemes for a network with the heterogeneous channel conditions used for the test case in Fig. 6 when the up-link capacity of each peer is $400 \mathrm{kbps}$. Because the random peer selection does not consider the packet loss of candidate child-peers, it leads to packet loss rates of higher than 0.8 under all channel packet drop rates, resulting in very poor video quality. The PS-T-model method reduces the packet loss rate to 0.077 for the channel condition with $[0.01,0.05]$ channel packet drop rate but still leads to a high packet loss rate of 0.663 under $[0.01,0.1]$ channel drop rate. In contrast, the proposed PS-M-model method effectively mitigates the packet loss rate to 0.062 under $[0.01,0.1]$ channel drop rate. Under the same packet drop rate settings, the PS-M-model method can provide more reliable video streaming services because the parentpeers are able to efficiently utilize the uplink bandwidth based on the accurate M-model. Nevertheless, both PS-M-model and PS-Tmodel cannot achieve reliable visual communication when the packet drop rate reaches $[0.01,0.2]$ since the effective uplink bandwidth capacity of peers cannot afford the redundancy of $F E C(6,4)$ under such a poor channel condition.

\section{CONCLUSION}

In this paper, we proposed an accurate model to estimate the packet loss probability in a mesh-based P2P network. The proposed model takes into account the channel packet drop rate, peer dynamics, and FEC protection to capture the heterogeneous packet loss behavior of individual video substreams transmitted over the irregular transmission paths of a mesh network. We have shown that, based on the proposed model, the parent-peers can appropriately select their child-peers to effectively mitigate the error propagation due to the packet losses that occur in their candidate child-peers. Our simulation results show that the proposed peer selection method effectively reduces the packet loss probability in a mesh-based network.

For the pull-based streaming systems, the child-peers schedule to request individual video packets of a video substream rather than the whole substream. As a result, one video substream can be received from multiple parent-peers. We can adopt the proposed packet loss models for a substream to estimate the packet loss probability for a pull-based streaming system. However, the parent-peers in pull-based streaming systems cannot efficiently allocate their uplink bandwidth. Therefore, we propose a pushpull-based streaming system in which the parent-peers can select appropriate child-peers to reduce their packet loss probability before pushing the video substreams.

\section{REFERENCES}

[1] Y. Shan, I. V. Bajić, S. Kalyanaraman, and J. W. Woods, "Overlay multi-hop FEC scheme for video streaming," Signal Process.: Image Commun. vol. 20, no. 8, pp. 710-727, 2005.

[2] B. Akbari, H. R. Rabiee, M. Ghanbari, "Packet loss in peerto-peer video streaming over the Internet. Multimedia Systems. pp. 345-361, 2008.

[3] E. Setton, P. Baccichet, and B. Girod, "Peer-to-peer live multicast: A video perspective," Proc. IEEE, vol. 96, no. 1, pp. 25-38, Jan. 2008.

[4] P.-J. Wu, J.-N. Hwang, C.-N. Lee, C.-C. Gau, and H.-H. Kap "Eliminating packet loss accumulation in peer-to-peer streaming systems," IEEE Trans. Circuits Syst. Video Technol., vol. 19, no. 12, pp. 1766-1780, Dec. 2009.

[5] S. Xie, B. Li, G. Y. Keung, and X. Zhang, "Coolstreaming design, theory, and practice. IEEE Trans. Multimedia. vol. 9, no. 8, pp. 1661-1671. Dec. 2007.

[6] PPStream [Online]. Available: http://www.ppstream.com

[7] PPLive [Online]. Available: http://www.pplive.com

[8] P2Pstrsim [online]. Available: http://media.cs.tsinghua.edu.cn/ zhangm/

[9] ISO/IEC JTC1/SC29/WG11 and ITU-T SG16 Q.6, 2007. 